Revue d'histoire de l'Amérique française

REVUE D.HISTOIRE DE L'AMÉRIQUE FRANÇAISE

\title{
Un document inédit sur l'Île de Sable et le Marquis de la Roche
}

Joseph Le Ber

Volume 2, numéro 2, septembre 1948

URI : https://id.erudit.org/iderudit/801451ar

DOI : https://doi.org/10.7202/801451ar

Aller au sommaire du numéro

Éditeur(s)

Institut d'histoire de l'Amérique française

ISSN

0035-2357 (imprimé)

1492-1383 (numérique)

Découvrir la revue

Citer cet article

Le Ber, J. (1948). Un document inédit sur l'île de Sable et le Marquis de la Roche. Revue d'histoire de l'Amérique française, 2(2), 199-213.

https://doi.org/10.7202/801451ar d'utilisation que vous pouvez consulter en ligne.

https://apropos.erudit.org/fr/usagers/politique-dutilisation/ 


\section{UN DOCUMENT INÉDIT SUR L'ILE DE SABLE ET LE MARQUIS DE LA ROCHE}

Le document que nous publions est un accord passé le 21 Février 1603 devant les tabellions de Rouen, entre le Marquis de la Roche et un Capitaine de Navire appelé Thomas Chefd'hostel.

Le Marquis de la Roche! Un homme animé des meilleures intentions, roulant dans sa tête les plus beaux projets, qui aurait pu avant Champlain, jouer un rôle décisif dans la colonisation de la Nouvelle-France et qui, dans toutes ses entreprises fut poursuivi par une malchance inouie.

C'était un ancien page de la reine Catherine de Médicis, laquelle, sans doute, lui fit octroyer par le Roi Henri III, une commission pour coloniser le Canada.

Cette vieille reine, dont on n'a su dire que du mal, était une grande politique et certains de ses desseins étaient vastes... comme l'Amérique. L'un, visant à prendre possession du Brésil, resta secret et fut inconnu jusqu'aux découvertes faites par $M$. de la Roncière, l'historien de la Marine Française ${ }^{1}$ : Philippe Strozzi, son cousin, devait en être le vice-roi. Il n'alla pas au-delà des Açores où il fut vaincu et tué par les Espagnols.

L'autre projet devait reprendre, sur une plus vaste échelle, semble-t-il, la tentative de Jean Ribault en Floride: à son ancien page Troïlus du Mesgouez, Marquis de la Roche, la reine fit donner commission par le Roi Henri III, en mars 1577, pour " s'appatrier aux TerresNeuves ) de l'Amérique du Nord et " faire siennes toutes et chacune les terres dont il se pourra rendre maître ). Le 3 janvier 1578, le titre de vice-roi ès dites Terres-Neuves, lui était conféré; sa juridiction devait s'étendre sur toutes les côtes, ou presque, de l'Atlantique

1. Ch. de la RoncIère, Histoire de la Marine Française. T. IV, p. 167 et sq "Le Secret de la Reine". 
Nord. Instruit par les récits des pêcheurs bretons sur les possibilités d'établissement en Amérique, Troillus du Mesgouez prépara une expédition pour prendre possession de son royaume. Hélas! Il n'alla pas loin; l'Angleterre qui déja avait des visées sur Terre-Neuve, lui barra la route: à peine sorti du port son vaisseau fut pris par quatre bâtiments britanniques, tandis que sir Humphrey Gilbert s'en allait prendre pied dans la grande île... pour peu de temps il est vrai, car au retour il se perdit sur l'île de Sable où il resta deux ans, dit Champlain.

Têtu comme un Breton qu'il était, Troïlus s'obstina: en 1584, avec le concours d'armateurs basques et malouins, il voulut renouveler sa tentative et prit à son bord 300 colons et des missionnaires. Peine perdue! son vaisseau échoua non loin de Brouage où il était venu embarquer du sel pour sa pêche ${ }^{2}$.

Bientôt la guigne le poursuivit sur un autre terrain. C'était l'époque des Guerres de Religion; le Marquis de la Roche, fidèle royaliste, prit parti pour Henri IV, mais un des chefs de la Ligue, le duc de Mercœur, le fit prisonnier et pendant sept longues années, il fut retenu dans les prisons de Nantes. Ses loisirs forcés, il les employa sans doute à se documenter sur son lointain royaume et à méditer sur les moyens propres à s'en assurer un jour la possession, car, à peine libéré, dès novembre 1596, nous le voyons à Honfleur préparant une nouvelle expédition. Henri IV qui savait récompenser ses fidèles, surtout s'ils étaient malheureux, lui renouvelle alors son ancienne commission et, bientôt, le 16 février 1597, le Marquis de la Roche conclut un accord avec le Capitaine de navire Thomas Chefd'hostel, en vue de conduire à l'île de Sable une compagnie de soldats sous le commandement de trois officiers ${ }^{3}$. Quels étaient ces soldats? Sans doute des chômeurs, mendiants et vagabonds que le Roi lui permit d'embaucher. C'était l'usage alors de faire des soldats et des colons avec les va nu-pieds: nous en avons d'autres exemples, soit pour les Antilles, soit, en particulier, pour la Guyane lorsque Le Roux de Royville, puis Poncet de Bretigny essayèrent d'y fonder une colonie. D'ailleurs on trouve des arrêts du Parlement qui, de temps en temps, enjoignent aux vagabonds de quitter la province "sous peine de la

2. Id. p. 312.

3. Sté d'Histoire de Normandie. Documents relatifs à la Marine Normande, par Charles et Paul BreARD: p. 60 et sq. (Rouen, Lestringant, 1889). 
vie ". On comprend que ceux-ci n'hésitaient pas alors à se laisser racoler pour de lointains voyages.

Pourquoi faire choix de l'île de Sable, ilot perdu en pleine mer, battu par tous les vents, loin des côtes, quasi dépourvu de végétation? Ça ne paraît pas très indiqué pour fonder une "habitation "! C'est que de là, pensait-il, il commanderait les vastes territoires de "Canada, Hochelaga, Terre-Neuve, Labrador, Noronbègue, Saguenay et autres pays adjacents " qui lui étaient échus! Dans le langage de l'époque, les vieilles cartes en témoignent, ces territoires s'étendaient depuis le 40e parallèle environ, jusqu'au cercle polaire, et l'île de Sable forme au centre une place avancée qui, fortifiee, permettrait de surveiller les côtes de l'Amérique du Nord, et d'arrêter au passage tous ceux qui se mêleraient de commercer aux Terres-Neuves sans son autorisation, comme les Lettres Patentes du 12 janvier 1598 lui en donnaient le droit.

A son retour de cette première expédition, Chefd'hostel fit un rapport qui dut être concluant sur l'avenir d'une colonie à l'île de Sable et le Marquis de la Roche continua son recrutement; en plus des mendiants et chômeurs, il réclama plusieurs fois au Parlement de Rouen des condamnés de droit commun comme un édit du Roi, disait-il, lui permettait de le faire; il est possible qu'il en ait embarqué quelques-uns, mais il semble bien, d'après ce que raconte Gosselin ${ }^{4}$, qu'abusant du pouvoir à lui octroyé par le Roi, il trouva plus avantageux de faire payer la forte somme aux criminels de bonne famille et de leur rendre la liberté.

Gosselin pense qu'il ne partit pas pour son royaume avant janvier 1599, parce que les registres du Parlement au 18 mai et au 15 octobre 1598 et à d'autres dates encore peut-être, mentionnent ses demandes de prisonniers, et qu'à son sens il n'aurait pas eu le temps d'aller au Canada et d'en revenir; or, les cinq mois compris dans cette période étaient largement suffisants, et au surplus le Marquis avait donné une procuration le 19 mars 1598 à un Sieur Martin Le Lou pour s'occuper de ses affaires et celui-ci pouvait fort bien le représenter devant le Parlement; habituellement, on a pu le constater maintes fois, les procurations étaient passées devant notaires, peu de jours avant le départ. Cette fois de la Roche avait frété deux navires:

4. Gosselin, Nouvelles Glanes historiques Normandes, (Rouen 1873) p.10 a 17. 
la Catherine commandée par le Capitaine Thomas Chefd'hostel, et la Fransoise sous les ordres du Capitaine Girot. Les chartes-parties en furent passées devant les tabellions de Honfleur les 16 et 18 mars $1598^{5}$. On estime que ce départ se fit vers la mi-avril et de la Roche était du voyage puisqu'un document du 17 octobre 1598 note qu'il était revenu " pour lever et mener personnes auc it pais "). ${ }^{6}$

Le voici donc parti pour l'Amérique. Pendant longtemps, sur la foi de Champlain, on a cru qu'arrivé à l'île de Sable, il n'y débarqua qu'une cinquantaine de colons; les autres refusant absolument d'y rester, il dut les ramener en France. Pendant cinq ans, dit-on, sept ans même selon Champlain, les colons débarqués dans l'île furent abandonnés par Troïlus de la Roche et c'est en 1603 seulement que, mis au courant de leur lamentable situation, le Parlement de Rouen, disent les uns, le Roi, disent les autres, intima au Marquis l'ordre de les envoyer chercher. On sait comment le Capitaine Chefd'hostel, chargé de cette mission, n'en trouva plus que onze dans le plus affreux dénuement, à demi-sauvages, se terrant dans des trous, vivant uniquement des poissons qu'ils pêchaient, ainsi que des bêtes sauvages et des loups marins qu'ils arrivaient à capturer et dont les peaux leur servaient de vêtements. Chefd'hostel les ramena en France; Henri IV les fit venir à Paris, leur remit les condamnations qu'ils avaient encourues et les gratifia même de cinquante écus chacun. Gosselin a raconté comment le Parlement de Rouen faisant droit à leur demande, leur accorda les deux tiers des peaux et huiles qu'ils avaient emmagasinées, alors que Chefd'hostel voulait garder le tout pour lui, sans doute pour les frais de leur retour.

Dans ce récit de l'abandon de cinquante forçats à l'île de Sable, il y a - nous allons le voir - un mélange de vrai et de faux dont la discrimination n'a pu être faite que de nos jours.

Dans un article paru en $1933^{7}$, M. Gustave Lanctot est venu prouver que les choses ne se sont pas du tout passées comme l'ont raconté Champlain et Lescarbot, suivis, sauf quelques variantes, par

5. BREARD. op. cit. p. 78-83.

6. Arrhives de la Seine-Inférieure - Registre de la Tournelle.

7. The Canadian Historical Association (Ottawa 1933) p. 33 et $8 q$. 
tous les historiens. Ses preuves sont tirées de deux pièces documentaires inédites jusqu'alors:

1o - La Commission de Henri IV au Capitaine Chauvin de Tonnetuit, retrouvée en 1926 sur la table d'un commissaire priseur de New-York, datée du 15 Janvier 1600;

2o - Une autobiographie écrite par le Marquis de la Roche luimême, vers 1604, dont quelques paragraphes apportent des lumières suffisantes pour démolir complètement la légende de l'abandon des colons à l'île de Sable .

D'après ce récit, voici comment les choses se seraient passées: Le Marquis de la Roche à peine arrivé à l'île de Sable change son nom en celui d'île de Bourbon, en l'honneur du Gouverneur de Normandie ${ }^{9}$; il y installe ses colons, fait bâtir des habitations et un magasin pour les vivres et approvisionnements de toutes sortes.

Vers la fin du mois d'août, après avoir laissé la colonie sous les ordres du Commandant Querbonyer et le magasin à la garde du Capitaine Coussez, il repart pour la France où il est de retour avant le 17 octobre. Il revoit Henri IV qui lui fait verser une subvention de douze mille écus.

Chaque année, sauf une, dit-il, il prit soin d'envoyer Chefd'hostel ravitailler sa petite colonie, ce qui prouve qu'il ne l'abandonnait pas comme on l'a dit. Mais, on ne sait pourquoi " les mendiants colons se révoltèrent une première fois et massacrèrent pendant son sommeil le Commandant Querbonyer "), puis ils assassinèrent le garde magasin Coussez, et plusieurs autres après lui. M. Lanctot pense que ces faits se produisirent en 1602 et " qu'en conséquence de cette situation... La Roche cessa d'expédier des provisions puisque son autorité avait

8. Escrit envoyé par le Marquis Troille du Mesgouez de la Roche Mescouez en Bretagne au Roy Henri IV environ l'année 1596 ou 1597 (ou plutôt 1598 ou 1599) lors de la Paix avec le duc de Mercœur sur le subject principalement de la traverse et contestation qui luy estait faite dans l'Isle de Bourbon a vingt cinq lieues de distance du Cap Breton vers la Nouvelle France et la Floride en l'Amérique, dans la Baye le long de la rivière vers Cadessart, dont la possession luy avait esté donnée par Henri III et IV (Bibliothèque de l'Institut, Collection Godefroy. Vol. 291. Fol. 149).

9. Le Gouverneur de Normandie, à cette époque, était bien un Bourbon, mais on l'appelait le duc de Montpensier! Nous aurions cru plutôt que le Marquis avait donné ce nom de Bourbon à son île soit en l'honneur du Cardinal Charles de Bourbon, archevêque de Rouen qui, jadis, avait financé avec l'amiral de Joyeuse son expédition manquée de 1584, soit pour faire sa cour à Henri IV le premier des rois Bourbons; mais si lui-même le dit, nous devons l'en croire. Il serait curieux alors de savoir quels liens l'unissaient au duc de Montpensier. 
disparu par le meurtre de ses lieutenants "), de sorte qu'au printemps de 1603, ils ne reçurent pas le ravitaillement habituel et tombèrent dans un dénuement complet. Cette hypothèse de $M$. Lanctot était tout à fait plausible; mais le document que nous avons relevé dans le tabellionage de Rouen vient la modifier et apporter un peu de lumière sur le drame de l'île de Sable; ce document, avons-nous dit, est un accord entre le Marquis de la Roche et le Capitaine Chefd'hostel, pour un nouveau voyage à l'île de Sable. Or, c'est précisément à la suite de ce voyage que les derniers colons de l'île furent ramenés en France. En analysant ce document, tâchons maintenant d'en tirer quelques renseignement nouveaux.

Messire Troïlus du Mesgouez commence par décliner ses titres et qualités et entre autres ceux de " Gouverneur et Lieutenant pour Sa Majesté des Terres Neufves de la Grand'Baie, Bacalan, Cap Breton, Ochelaga, Tadosacq, Isle de Bourbon et terres adjacentes. ")

Si nous les comparons à ceux qu'il se donnait en 1598 lors de ses accords avec les capitaines Chefd'hostel et Girot ${ }^{10}$, nous y trouvons quelques variantes: dans ces derniers, il se qualifiait " lieutenant général pour le roy en pays de Canadas, isle de Sable, Terre-Neuve, Labrador, Hochelagua, Saguenay et autres pays adjacents "). A part Ochelaga, la nomenclature des terres de son vice-royaume ne parait plus la même, mais en réalité elle désigne les mêmes régions, les TerresNeuves de la Grand'Baye signifiant tous les territoires entourant la baie du St-Laurent qui sont ensuite énumérés: Bacalan est un point sur la côte de Terre-Neuve, dans la zone qui fut portugaise, mais c'est aussi d'après les cartes du XVIème siècle, Terre-Neuve, le Labrador, l'Acadie; c'est tantôt une île, tantôt une péninsule ou même une partie du continent ${ }^{11}$; le Cap Breton pouvait désigner toute l'Acadie; Ochelaga, non seulement le village indien de ce nom devenu depuis Montréal, mais une immense région du Canada. L'île de Bourbon, nous le savons maintenant, n'est autre que l'íle de Sable. Il n'est plus question de Saguenay, vaste territoire au nord du St-Laurent, mais d'un point de ce territoire: Tadoussac. Nous sommes en 1603, or le poste de Tadoussac venait d'être fondé en 1600, par Pierre

10. Breard, op. cit. p. 80.

11. L'abbé Anthinume, Cartes Marines (Paris, Dumont 1916) T. 11, p. 507. Note 8. 
Chauvin, Seigneur de Tonnetuit. Comme on le voit par ce détail, le Marquis de la Roche n'ignorait pas que son rival avait établi une habitation en cet endroit, et il continuait a revendiquer le gouvernement de toute la région, conforménemt aux Lettres-patentes du 15 janvier 1600 analysées par M. Lanctot dans son article, ${ }^{12}$ lettres, dit-il, qui établissent " que Chauvin avait précédemment obtenu une commission de Henri IV dès l'année 1599 ), mais qui " maintiennent l'autorité vice-royale de La Roche sur les Terres-Neuves 》 et " accordent à Chauvin... le droit de représenter le vice-roi et d'exercer tous ses pouvoirs... $)^{13}$

Il est question aussi des " Commissions qu'il dit luy avoir esté données par le Roy "..., malheureusement, il n'a pas da les présenter au tabellion, pas plus que la Lettre de Sa Majesté lui commandant cette nouvelle expédition, de sorte que l'acte n'en donne pas la date.

On a prétendu que Chefd'hostel avait entrepris son voyage sur l'ordre du Parlement ou du Roi, ou encore par simple humanité. On ne voit rien de semblable dans cet acte! Que dit-il, en effet? Que Chefd'hostel doit cingler vers l'île de Bourbon avec son navire " $L a$ Catherine " du port de 140 tonneaux, attaché pour lors aux quais de la Ville Françoise de Grâce, autrement dit Le Havre. Il y transportera un "commissionnaire " ou fondé de pouvoirs dont le nom n'est pas spécifié; et là tous deux visiteront le capitaine Carbonnier, ${ }^{14}$ lieutenant du Marquis en ladite île, et les soldats qui y sont sous ses ordres, soldats dont les noms, au moins pour quelques-uns lui sont connus et à qui il permet de revenir au nombre de huit ou dix au maximum, ce sont, outre le Capitaine Carbonnier, un gentilhomme de sa suite appelé le Cadet de la Touche, parent peut-être de Daniel de la Touche, sieur de la Ravardière qui tentera peu après de s'établir à la Guyane; Coussy Bretonnière, qu'on peut identifier avec le garde magasin Coussey, Regnardière, Lamare et le Parisien. Tous ceux-ci pourront rapporter leurs coffres et leurs bagages. Ce fait permet de

12. The Canadian Historical Association, p. 37.

13. Ceci nous explique pourquoi dans une transaction passée devant les tabellions de Rouen le 23 février 1602, entre les marchands de St-Malo et Pierre Chauvin, il est dit que celui-ci avait été " estably du Sieur Marquis de la Roche au pays de Canada ".

14. Sans nul doute, le Commandant Querbonyer mentionné dans l'x Escrit...» 
supposer que la colonie avait eu primitivement des cadres assez importants puisque de la Roche juge pouvoir sans inconvénients la priver au moins pendant un an de ses principaux chefs. Il donne au porteur de sa commission et à Chefd'hostel l'ordre de " regarder au plus près ladite île ", le nombre de bêtes à cornes et de porcs qu'elle renferme, d'examiner la fertilité du sol, noter ce qui est nécessaire pour l'exploiter et les plantations qui lui conviennent. Il faut avouer que cette ignorance des possibilités de l'île après cinq ans d'établissement, est bien surprenante et cela laisse entrevoir que Troillus s'en était passablement désintéressé! Il ajoute qu'ils devront conférer de tout cela avec le Sieur Carbonnier et les gens de sa suite; leur rapport est nécessaire car, dit-il, Sa Majesté veut savoir de source certaine si on peut fonder dans cette île une habitation sûre et commode.

Quant aux peaux, cuirs, huiles et autres marchandises emmagasinées par les colons dans l'île de Bourbon, Chefd'hostel en chargera dans son navire la quantité que Carbonnier déterminera, et pour le fret, Chefd'hostel, ses bourgeois, mariniers et tiercements auront les deux tiers de ces marchandises et l'autre tiers reviendra au Marquis de la Roche, comme le portaient déjà les charte-parties de 1598.

Les autres clauses du contrat ont moins d'importance pour nous: comme toujours, en pareil cas, Chefd'hostel pourra se livrer à la pêche à ses dépens et il en aura le profit, car la pêche parait bien être le but de son voyage et le Marquis, au courant de son projet, a saisi cette occasion pour faire prendre par cet homme en qui il a grande confiance des renseignements sur l'île de Bourbon et pour donner une permission de détente à quelques colons.

Quant aux victuailles et frais du voyage, il semble ressortir du texte qu'ils seront à la charge de Chefd'hostel pour l'aller, sauf dans une certaine mesure dont ils ont convenu ensemble; mais pour le ravitaillement du retour, le Marquis de la Roche permet qu'on prenne dans l'île trente bœufs et vingt pourceaux mâles " sans point prendre aucune femelle ", ce qui se comprend parfaitement pour ne point faire tort à la reproduction; Chefd'hostel aura encore les deux tiers sur les peaux de ces bœufs-là, et le Marquis de la Roche le tiers, comme pour toutes celles qui seront ramenées de l'île.

Essayons maintenant à l'aide des derniers documents connus d'imaginer le déroulement des événements depuis l'arrivée des colons à l'île de Sable en 1598 . 
Après les avoir installés avec des commodités suffisantes, pense-til, Troillus du Mesgouez rentre en France et continue son recrutement. En 1599 son fidèle ami Chefd'hostel emmène encore quelques hommes avec des " rafraichissements en vins, habits et hardes ".

En 1600 et 1601, nouveaux voyages où Chefd'hostel fait encore escale à l'île de Sable ou de Bourbon, peut-être avec un peu de ravitaillement mais surtout pour prendre des nouvelles et charger les peaux et huiles amassées pendant l'année. On doit avouer que ce ravitaillement " en vins, habits et hardes " était fort peu de choses, et très insuffisant pour une colonie naissante. Aujourd'hui, on sait que pendant une assez longue période, une colonie est incapable de se suffire à elle-même, mais en ce temps-là on pensait qu'après les premiers frais d'établissement, les colons pouvaient se tirer d'affaire tout seuls, et c'est à cause de cela que nos premières colonies se plaignirent toutes, à leurs débuts, de n'être pas suffisamment " rafraichies "), qu'il s'agisse du Canada, de St-Christophe, de la Guadeloupe ou de la Guyane. La même faute fut commise par le marquis de La Roche, il crut qu'au bout d'un an ou deux de séjour à l'île de Sable, ses colons feraient des récoltes assez abondantes pour tous leurs besoins alimentaires, c'est pourquoi il n'envoya que du vin et des habits, et même en 1603 il n'envoya sans doute aucun ravitaillement puisque notre document n'en souffle mot.

De plus, on doit le souligner, de la Roche était avant tout un soldat et si avec une troupe hétérogène de vagabonds, on arrive à faire des soldats, il ne paraît pas s'être douté qu'il faut des hommes de métier pour fonder et faire vivre une colonie; avec de tels éléments, les " jardinages " ne durent pas être très productifs, et les travaux de toutes sortes qui exigent des artisans laissèrent à désirer. Quoi d'étonnant alors que la colonie fut décimée par les privations, la famine et les épidémies, comme toutes celles du XVIIème siècle à leurs débuts, sauf peut-être le Canada dont les colons étaient d'une autre qualité.

Monsieur Lanctot suppose, avec raison, croyons-nous, que les mutineries eurent lieu en 1602, car c'est probablement cette année-là que de la Roche n'envoya personne visiter son établissement, non pas parce que ses lieutenants avaient été assassinés, - il ignorait encore le drame au début de 1603 puisqu'il parle d'en ramener le Capitaine Carbonnier et Coussy, supprimés depuis de longs mois par les mutins, - 
mais pour une autre raison qu'il est pour le moment impossible de préciser. Il en autorise encore quelques autres à revenir, mais ceux qu'il nomme étaient morts aussi, car aucun ne figure dans la liste des onze rescapés publiée par Gosselin ${ }^{15}$.

Il est probable que pris par d'autres soucis, peut-être par manque de ressources ou parce que son envoyé habituel le Capitaine Chefd'hostel n'était pas disponible, le Marquis de la Roche négligea en 1602 d'envoyer un navire visiter le poste. Et nous avons ainsi les quatre voyages faits en cinq ans par Chefd'hostel, soi-disant pour ravitailler là colonie: $1599-1600-1601$ et 1603 .

En 1602, n'ayant pas vu venir le messager habituel, les colons se crurent oubliés et abandonnés de leur chef lointain; souffrant de la faim, persuadés que leurs officiers avaient des provisions cachées, les anciens vagabonds menés peut-être par quelques galériens graciés venus en même temps qu'eux, se concertèrent et tuèrent leurs chefs pour s'emparer des vivres qu'ils croyaient en réserve dans le magasin confié à Coussy.

Dans son "Ecrit... " de la Roche prétend avoir ravitaillé l'île, nous avons vu qu'en somme c'était fort peut de chose; de plus, il serait imprudent de prendre son affirmation au pied de la lettre; cet "Ecrit", il ne faut pas l'oublier, est un plaidoyer "pro domo"); comme tous ceux qui sont menacés de perdre leurs privilèges, comme Guillaume de Caen pour le Canada, comme Jacob Bontemps pour la Guyane, de la Roche grossit ce qu'il a fait pour le bien de sa colonie, exagère ses frais et cherche à persuader qu'il a tenu tous ses engagements.

A cette époque, nous dit Champlain, le Marquis de la Roche était à la Cour et sans doute sollicitait-il de Sa Majesté une nouvelle commission de lieutenant général au Canada. Malgré l'amitié qu'il avait pour ce vieux serviteur, ancien combattant et martyr de sa cause, Henri IV avait dû être très mal impressionné par l'insuccès de ses précédentes tentatives, par sa malchance persistante et il voulait des garanties avant de lui renouveler sa confiance et de proroger ses pouvoirs; et puis ce pauvre Marquis avait des rivaux, rivaux animés de l'esprit d'entreprise... mais surtout d'entreprises commerciales,

15. Gosselin. Op. cit. p. 16. 
hélas! et qui, ajoute Champlain, " n'avaient désir que le vrai culte de Dieu s'accreust, ni d'y voir florir la religion catholique, apostolique et romaine. $)^{16}$. Cette phrase paraît bien désigner Chauvin qui était protestant et pensait toujours supplanter de la Roche comme lieutenant général, mais comme il mourut au début de 1603, de la Roche revient à la charge auprès du roi. Le roi circonvenu par les amis de Chauvin, hésitait donc; il nomma le Gouverneur de Dieppe Aymar de Chastes à la place de Chauvin et demanda à de la Roche des précisions sur les résultats obtenus depuis cinq ans; voilà pourquoi celuici envoya un " commissionnaire " à l'île de Sable chargé d'étudier avec soin ses ressources " affin que Sad. Majesté puisse estre certaine de l'abitation asseurée de lad. île et commodité d'icelle "; l se peut même que ce chargé de commission, dont le Marquis ne donne pas le nom, Henri IV se soit réservé de le désigner lui-même au dernier moment. Le Roi, en effet, ne cessait de s'intéresser à l'Amérique et en particulier au Canada, malgré son ministre Sully pour qui " labourage et paturage " valaient mieux que toutes les richesses du Pérou, c'est-à-dire du Nouveau Monde.

Ainsi donc, quoi qu'on en ait dit, le Marquis de la Roche n'a été condamné ni par le Parlement de Rouen, ni par le Roi à rapatrier les misérables abandonnés de l'île de Sable. Gosselin qui a feuilleté les registres du Parlement n'a pas trouvé trace de cet arrêt, et il attribue aux remords de la Roche l'envoi de Chefd'hostel pour ramener les survivants.

Il est probable que Chefd'hostel, lors de son arrivée à l'île de Sable, éprouva quelque surprise et que le porteur de la commission de Troïlus du Mesgouez exigea des explications et fit une enquête; puis on embarqua cette triste troupe, peut-être à fond de cale, comme des criminels, tels qu'on les trouva, vêtus de peaux de bêtes, avec des barbes de Neptune et les cheveux en désordre où le ciseau n'avait pas pénétré depuis plusieurs années. Ils durent faire sensation lorsqu'en cette tenue ils mirent pied sur les quais du Havre, puis de Rouen. Le Roi en eut bientôt connaissance et les fit venir à la Cour, par curiosité peut-être, mais surtout pour recevoir de leur bouche les renseignements qu'il désirait sur les possibilités d'un établissement

16. Voyages du Sieur de Champlain (Paris 1830) T. I. p. 42 et 43. 
durable à l'île de Sable. Ceux-ci craignant les plus grands châtiments à cause de leur révolte et des meurtres commis, ne minimisèrent sárement pas leurs souffrances et affirmèrent que depuis leur arrivée en l'île ils avaient été pour ainsi dire totalement abandonnés: pendant sept ans, dit Champlain! Et, en effet, si on admet que dès le voyage de 1597, des hommes de guerre y avaient été déposés avec le Capitaine Kerdement, le Lieutenant de Kéroual et l'Enseigne de Mondreville ${ }^{17}$, cela ne fait pas loin de sept ans. Le bon Roi Henri, ému par leurs récits, jugeant qu'ils avaient été laissés dans une situation inhumaine, sans secours d'aucune sorte durant plusieurs années, en proie à la misère et à la faim, toujours mauvaises conseillères, fut très indulgent et leur accorda des lettres de rémission pour leurs crimes; bien plus, il chargea même son argentier Sully de leur donner à chacun 50 écus. Naturellement, La Roche, qui aurait voulu " les faire pendre pour leurs malfaits " que d'eux-mêmes ils avaient avoués, fut fort indigné: Champlain revenu peu de temps auparavant de son premier voyage au Canada, eut peut-être l'occasion de voir de ses yeux ces tristes débris d'une colonie, ramenés par Chefd'hostel et d'entendre le récit de leurs malheurs, sinon de leur bouche, du moins de la bouche des ennemis du Marquis de La Roche; il demeura sur cette impression de sorte qu'écrivant trente ans après l'événement il ne se souvint que de cette vision de pauvres hères à moitie morts de faim et accepta sans contrôle des racontars sur des événements où lui-même n'avait pas été acteur. Nous savons depuis les dernières guerres comment se créent facilement des légendes, surtout lorsqu'elles sont voulues et ( dirigées ). Champlain, sans le vouloir, a contribué à répandre la légende d'un abandon total à l'île de Sable, que les ennemis de Troïlus de la Roche ne manquèrent pas de propager; et ce sont sans doute ces mêmes personnages qui poussèrent les rescapés à réclamer les peaux et huiles rapportées de l'île par Chefd'hostel; le Parlement de Rouen fut saisi de l'affaire et, dit Gosselin ${ }^{18}$, " faisant justice à tous, décida que Chefd'hostel aurait un tiers desdites peaux et que le reste serait partagé entre les onze hommes qu'il avait ramenés".

17. Bréard. Op. cit. p. 75-78.

18. Gosshurn. Op. cit. p. 17. 
On peut penser que s'ils se virent attribuer les deux tiers de ces marchandises, c'est parce que de la Roche n'avait rien fait pour eux depuis au moins deux ans et qu'on trouva juste de leur laisser ce qu'ils ne devaient qu'à leur seule industrie.

Gosselin a oublié de donner la référence de cet arrêt; nous avons eu la chance de le retrouver avant la guerre, nous réservant d'en prendre copie plus tard; malheureusement depuis lors et pour longtemps encore peut-être, il est impossible de consulter les Archives du Parlement ${ }^{19}$. Cet arrêt se trouve aux Registres d'audience le 27 Novembre 1603. Peut-être nous apporterait-il quelques détails intéressants sur cette affaire mémorable.

Quant au Marquis de la Roche, malchanceux jusqu'à la fin, il eut beau faire valoir tous les services rendus depuis quarante ans, il ne retrouva pas la faveur du Roi et mourut bientôt, découragé, ruiné peut-être, en l'année 1606 .

\author{
Joseph LE BER \\ curé de Veules-les-Roses (France)
}

19. L'humidité des locaux où elles sont déposées menace d'endommager gravement ces archives qui renferment tant de documents précieux pour l'histoire du Canada.

\title{
Document inédit sur la Roche et l'Ile de SABle
}

Du vendredy avant midy vingt et ung jour de febvrier mil six cent et trois passé en la maison et logis ou pend pour enseigne la croix blanche hors le pont de Rouen proche ledit pont.

Fut présent Messire Troïlus de Mesgouez chevallier de l'ordre du Roy Conseiller en son conseil d'Estat et privé, Cappitaine de cinquante hommes d'armes de ses ordonnances, gouverneur et lieutenant général pour Sa Majesté des Terres Neufves de la grand baye bacalan Ca breton Ochelaga Tadosacq Isle de Bourbon et terres adjacentes Sieur Marquis de la Roche, baron de lat Vicomte de Carentan et Saint Lô en Normandye et aussy vicomte de Trevallot demeurant en sa terre et seigneurie de la Roche gomart en bretagne, evesché de Cornouaille lequel suivant les commissions qu'il disoit luy avoir esté données par le Roy nostre Sire et lettre de Sa Majesté sur ce expédiée pour l'effect cy après: de son bon gré nommé commis et institué la personne de Thomas Chefd'hostel Cappitaine de navire demeurant en la parr. de Vasteville en la vicomté du Pont Audemer 
a ce présent et acceptant auquel portant ces présentes led. Seigneur Marquis de la Roche a donné pouvoir et puissance par icelles d'aller par led. Chefd'hostel pour luy ses bourgeois victuailleurs compaignons mariniers ot tiercemens du navire apartenant aud. Chefd'hostel a luy et ausdits bourgeois et consors nommé la Catherine du port sept vingt tonneaux ou environ estant de présent en la ville françoise de grâce en lad. Isle de Bourbon et aud. lieu y visiter par luy et par celluy commissionnaire que led. Sieur Marquis y envoyra dedans led. navire le cappitaine Carbonnyer lieutenant dud. Seigneur Marquis, ensemble les soldats estant en lad. Isle de Bourbon et regarder au plus près led. lieu le nombre qu'ils pourront juger des bestes à corne et pourceaux et la fertilité d'icelle Isle et d'adviser ce qui sera nécessaire en cedit lieu et quelle semence et fruits lad. Isle pourra porter en conférant du tout avec led. Sieur Carbonyer et gens de commandement de sa suite affin que Sad. Majesté puisse estre certaine de l'abitaon asseurée de lad. Isle et commodité d'icelle. Et pour cest effect led. Seigneur Marquis a permis aud. Chefd'hostel de rapporter dedans sond. navire led. cappitaine Carbonyer avec ung gentilhomme de sa suilte nommé le cadet de la touche Coussy Bretonnière, Regnardière, La mare, Le parisien et autres personnes que jugera led. cappitaine Carbonyer pourveu toutesfois qu'il n'en soyt admené avec led. cappitaine Carbonyer en plus avant que huit ou dix personnes oultre led. commissionnaire que yra avec led. Capp. Chef d'hostel, lesquels pourroient rapporter leurs coffres et baggages, et a esté expressément convenu et accordé entre led. Seigneur marquis et icelluy Chefd'hostel pour luy sesd. bourgeois et consors mariniers et tiercemens que icelluy Chef d'hostel pour luy et ésdits noms auront les deux tiers des cuirs peaulx et huilles et autres marchandises qui se trouveront en lad. Isle que led. Chefd'hostel y prendra et chargera dedans sond. navire par le commandement et ordre dud Sieur Carbonyer ov de ceulx qui auront de luy charge, et led. Seigneur Marquis l'autre tiers du tout franchement venant à son proffit. Le tout qui sera ainsy party entre eulx au retour du voyage dud. navire qui sera fait en lad. ville françoise de grâce; pour aller faire lequel voyage led. Chef d'hostel pourra prendre du sel ou bon luy semblera pour ce qu'il aura de besoing à faire sond. voyage et pesche aux frais et despens de luy Chef d'hostel parce que tout (ce) qui en proviendra sera chargé dedans led. navire et demeurera au proffit dud. Chefd'hostel pour luy et consors réservé led. tiers et ce qui sera prins en lad. Isle qui appartiendra comme dit est aud. Sieur Marquis sans qu'il soit tenu contribuer en aucune chose et frais comme dessus soyt pour luy et gens de compagnie ou autres despens ou autrement. A quoy led. Chefd'hostel pour luy et consors etc y demeurera subject. Et pour les victuailles tant dud. commissionnaire que dud. capp. Carbonyer et des gens qui reviendront de lad. isle dedans led. navire. Led. Seigneur Marquis sera tenu fournir desd. victuailles comme il est dit et ont convenu et accordé ensemble. Et sy a permis led. Seigneur Marquis aud. capp. Chefd'hostel de prendre et faire tuer en lad. Isle de Bourbon trente bœufs et vingt pourceaulx masles sans 
point prendre aulcunes femelles et la chair d'iceulx de la prendre pour la nourriture et victuailles de ceulx qu'il ramènera de lad. Isle et matelots et gens de son esquipage et ce oultre lesd. victuailles à quoi leur est tenu led. Seigneur Marquis comme dit est. Et pour le regard des pertes ${ }^{1}$ desd. trente bœufs ils seront payés aussy les deux tiers par led. Chefd'hostel pour luy et consors et l'autre tiers franches au proffit dud. Seigneur Marquis suivant l'accord que led. Seigneur Marquis et led. Chefd'hostel en disent avoir faict entre eux parce que lesdits Seigneur Marquis et Chefd'hostel pour luy et consors seront tenus courir les risques de la mer et de la guerre durant lesd. voyages lesd. périls et fortunes de la mer. Desquelles choses cy-dessus lesd. Seigneur Marquis et Chefd'hostel pour luy et consors etc. ont promis tout ce que dessus de tenir et entretenir suivant sa force et teneur esd. etc. et qualités chacun en leur regard de tous leurs biens et héritages. Présents Thomas Ravend escuier Sieur du bourg demeurant en la paroisse Saint Thénye ${ }^{2}$ en la vicomté de Carentan, baillage de Costentin et Toussaint Dupré delestre chirurgien demeurant en la paroisse Saint-Sever les Rouen au logis de Michel Delabarre Maître Chirurgien.

\section{Signé: TROILUS DU MESGOUEZ LA ROCHE CHEFDOSTEL, RAVEND, DUPRE.}

Nous remercions sincèrement Mlle M.-J. Le Cacheux, archiviste-paléographe, qui nous a beaucoup aidé au déchiffrement assez laborieux de cet acte.

J. L.-B.

1. Le mot étant très mal écrit, on peut lire aussi bien " peaulx ".

2. Pour Saint-Eny, près de Carentan, Manche.

Civilisation CANAdienNe-Francaise. - Nous publions dans ce no de la Revue, Un cours de civilisation canadienne-française, article de l'un de nos membres-correspondants, Mlle Marine Leland. Les lignes qui vont suivre et que nous empruntons à la Revue de l'Université Laval, renseigneront nos lecteurs, s'il en est besoin, sur l'opportunité d'un tel cours et sur le mérite de Mlle Leland, véritable initiatrice en ce domaine.

(I Il est évident que toute Université digne de ce nom devra bientôt offrir un cours, au Canada, sur la civilisation et la littérature canadiennesfrançaises aux points de vue: (1) social, (2) politique, (3) littéraire, (4) religieux; je dis religieux car, à mon avis, il est possible de s'approcher de ce sujet qui est, comme je l'ai dit au début, inséparable de l'éducation. A mon humble avis,sı l'on veut enseigner un cours sur le Canada français, dans une Unıversité, on ne peut le faire seulement au point de vue littéraire. Si le professeuः n'a pas suivi les cours d'une Université canadienne-française, il est fort probable que ses bonnes intentions feraient plus de mal que de bien, au Canada ))

(Extrait de La Revue de l'Université Laval, Québec, juin 1948, vol. II - no 10. "A la recherche de la vérité canadienne " par Frédéric J. L. Bronner, Hamilton, Ont.) 